\title{
On the Spaces of Self Homotopy Equivalences for Fibre Spaces II
}

By

\author{
Tsuneyo YAMANOSHITA*
}

\section{Introduction}

Let $X$ be a connected $C W$ complex with non-degenerate base point $x_{0}$. And let $G_{0}(X)$ be the space of self homotopy equivalences of $\left(X, x_{0}\right)$.

The purpose of this paper is to study $G_{0}(E)$ when $E$ is a fibre space of a fibration with fibre $K(G, n)(n>1)$ :

$$
K(G, n) \stackrel{i}{\longrightarrow} E \stackrel{p}{\longrightarrow} B
$$

If a base space $B$ is simply connected, we had some results on $G_{0}(E)$ in the previous papers $[16,17,18,19]$. Here we treat $G_{0}(E)$ for the case of a nonsimply connected base space $B$.

Let $G$ be an abelian group and let $\operatorname{Aut}(G)$ be its group of automorphisms. Denote by $L(G, n+1)$ the classifying space for fibrations with fibre $K(G, n)$ and by $W$ an Eilenberg-MacLane complex $K(\operatorname{Aut}(G), 1)$. Then we have the fibration:

$$
K(G, n+1) \stackrel{i_{0}}{\longrightarrow} L(G, n+1) \stackrel{p_{0}}{\longrightarrow} W .
$$

Under these notations our main results (Theorem 3.3, 4.4 and 4.7) are stated as follows.

Theorem 3.3. Let $X$ be a $C W$ complex, $k$ be a fixed map of $\left(X, x_{0}\right)$ to $\left(L(G, n+1), l_{0}\right)$ and $p_{0} \circ k=k^{\prime}:\left(X, x_{0}\right) \rightarrow\left(W, w_{0}\right)$ be a space over $\left(W, w_{0}\right)$. Then the space $\operatorname{map}_{0}(X, L(G, n+1))_{w}$ of maps over $\left(W, w_{0}\right)$ has the same weak homotopy type as

$$
H^{n+1}\left(X, x_{0} ; G\right) \times \prod_{i=1}^{n} K\left(H^{n+1-i}\left(X, x_{0} ; G\right), i\right)
$$

where the cohomology is taken with local coefficients classified by the map $k^{\prime}: X \rightarrow$ $W=K(\operatorname{Aut}(G), 1)$.

Communicated by N. Shimada, June 6, 1985.

* Department of Mathematics, Musashi Institute of Technology, Tamazutsumi, Setagaya, Tokyo 158, Japan. 
Denote by $\mathcal{G}(E \bmod F)$ the space of self fibre homotopy equivalences of $E$ leaving a fibre $F$ fixed in a fibration: $F \stackrel{i}{\longrightarrow} E \stackrel{p}{\longrightarrow} B$. We denote by $X \underset{w}{\widetilde{w}} Y$ when $X$ has the same weak homotopy type as $Y$. Then, by using the result proved in $[18,19]$ we have

Theorem 4.4. Let $p: E \rightarrow B$ be a fibration with fibre $F=K(G, n) \quad(n>1)$ such that $B$ is a CW complex. Then if we denote by $k:\left(B, b_{0}\right) \rightarrow\left(L(G, n+1), l_{0}\right)$ a corresponding map to the fibration: $F \stackrel{i}{\longrightarrow} E \stackrel{p}{\longrightarrow} B$, we have

$$
\mathcal{G}(E \bmod F) \underset{w}{\widetilde{w}} \operatorname{map}_{0}(B, L(G, n))_{W} .
$$

Let $\varepsilon(X)$ denote the group $\pi_{0}\left(G_{0}(X)\right)$ for a $C W$ complex $X$. Then we have the following theorem which is a generalization of Theorem 10 in [18].

Theorem 4.7. For a given $1 \leqq m<n$, let

$$
F=K(G, n) \stackrel{i}{\longrightarrow} E \stackrel{p}{\longrightarrow} K(\pi, m)=B
$$

be a fibration with a corresponding map $k:\left(B, b_{0}\right) \rightarrow\left(L(G, n+1), l_{0}\right)$. Then we have

$$
G_{0}(E) \underset{w}{\simeq} R \times H^{n}(B, G) \times \prod_{i=1}^{n-1} K\left(H^{n-i}\left(B, b_{0} ; G\right), i\right)
$$

where $R$ is the subgroup of $\operatorname{Aut}(\pi) \times \operatorname{Aut}(G)=\varepsilon(B) \times \varepsilon(F)$ consisting of $([g],[h])$ with

$$
\left[\chi_{\infty}(h)\right] \cdot[k]=[k] \cdot[g],
$$

and the cohomology is taken with local coefficients classified by the map $p_{0}^{\circ} k$ : $B \rightarrow K(\operatorname{Aut}(G), 1)=W$.

Thus as a corollary of Theorem 4.7 we have the following theorem $[9,11,15]$.

Theorem 4.8. Under the same hypothesis of Theorem 4.7 there exists the following exact sequence

$$
1 \longrightarrow H^{n}(B, G) \longrightarrow \varepsilon(E) \longrightarrow R \longrightarrow 1,
$$

where $R$ is the same group as the group stated in Theorem 4.7 and the cohomology is taken with local coefficients classified by the map $p_{0} \circ k: B \rightarrow K(\operatorname{Aut}(G), 1)$.

\section{Acknowledgement}

The author was stimulated by conversation with Mr. Y. Hirashima to start this work and heartily wishes to thank him. 


\section{$\S 1$. Fibrations}

Throughout this paper, we shall work within the category of compactly generated Hausdorff spaces [13] and by a base point we mean a non-degenerate base point.

Let $X$ and $Y$ be spaces with base points $x_{0}$ and $y_{0}$ respectively. The space of maps of $X$ to $Y$ will be denoted by $\operatorname{map}(X, Y)$ and $\operatorname{map}_{0}(X, Y)$ will be the subspace of $\operatorname{map}(X, Y)$ of maps of $\left(X, x_{0}\right)$ to $\left(Y, y_{0}\right)$. Moreover, when $k$ is a map of $X$ to $Y$, we denote by $\operatorname{map}(X, Y ; k)$ the path component of $k$ in $\operatorname{map}(X, Y)$, and $\operatorname{map}_{0}(X, Y ; k)$ is defined similarly.

Furthermore, throughout this paper a $C W$ complex means a connected $C W^{\top}$ complex with base point, unless otherwise stated.

Let $k:\left(X, x_{0}\right) \rightarrow\left(B, b_{0}\right)$ and $k^{\prime}:\left(Y, y_{0}\right) \rightarrow\left(B, b_{0}\right)$ be spaces over $\left(B, b_{0}\right)$, then we denote by $\operatorname{map}_{0}(X, Y)_{B}$ the subspace of $\operatorname{map}_{0}(X, Y)$ of maps over $\left(B, b_{0}\right)$ of $k$ to $k^{\prime}$. That is, each element $f$ of $\operatorname{map}_{0}(X, Y)_{B}$ satisfies $k^{\prime} \circ f=k$,

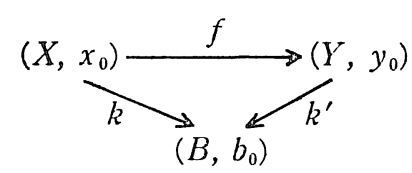

Let $p: E \rightarrow B$ be a space over $B$ and let $X$ be a space. We denote by $E_{B}^{X}$ the space of maps each of which is a map of $X$ to $E$ such that its composition with $p$ is a constant map of $X$ to $B$. Then the following diagram is commutative:

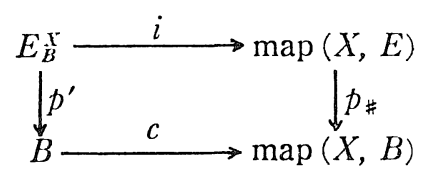

where $p_{\#}: \operatorname{map}(X, E) \rightarrow \operatorname{map}(X, B)$ is the map induced by $p_{2} p^{\prime}: E_{B}^{X} \rightarrow B$ is defined by $p^{\prime}(f)=p \circ f(x), c$ is a map defined by

$$
c(b)(x)=b \quad(b \in B, x \in X)
$$

and $i$ is the inclusion map.

Let $p: E \rightarrow B$ be a map and let $X$ be a space in the category. Then we say that $p$ is a fibration, if and only if it has the homotopy lifting property with respect to every $X$. Thus our fibration $p: E \rightarrow B$ is not necessary surjective.

Note that $p^{\prime}: E_{B}^{X} \rightarrow B$ is a fibration if $p: E \rightarrow B$ is a fibration. Let $X$ be a space with base point $x_{0}$. Define a map $\omega: \operatorname{map}(X, E) \rightarrow E$ by $\omega(f)=f\left(x_{0}\right)$. The restriction of $\omega$ on $E_{B}^{X}$ will be denoted by the same $\omega$, then we have the following 
Proposition 1.1. With the above notations, $\omega: E_{B}^{X} \rightarrow E$ is a fibration and the following diagram is commutative:

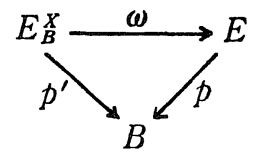

Proof. Define a map $\bar{p}: \operatorname{map}(X, E) \rightarrow E \times \operatorname{map}(X, B)$ by

$$
\bar{p}(f)=\left(\omega(f), p_{\#}(f)\right) .
$$

Then we can easily see that the following diagram is commutative:

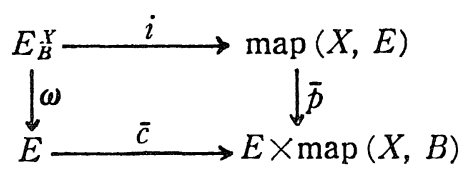

where $\bar{c}: E \rightarrow E \times \operatorname{map}(X, B)$ is defined by

$$
\begin{aligned}
& \bar{c}(e)=\left(e, c_{p(e)}\right), \\
& c_{p(e)}(x)=p(e) \quad(e \in E, x \in X) .
\end{aligned}
$$

Since $\bar{p}: \operatorname{map}(X, E) \rightarrow E \times \operatorname{map}(X, B)$ is a fibration (see Theorem 10 in [14]) and $\bar{c}$ is injective, we see that $\omega: E_{B}^{X} \rightarrow E$ is a fibration.

The equality $p \circ \omega=p^{\prime}$ follows immediately from the definition $\omega, p$ and $p^{\prime}$.

Remark 1.2. In Proposition 1.1, when a fibration $p: E \rightarrow B$ has a crosssection $s: B \rightarrow E$, the fibration $p_{\#}: \operatorname{map}(X, E) \rightarrow \operatorname{map}(X, B)$ has also a cross-section $s_{\#}: \operatorname{map}(X, B) \rightarrow \operatorname{map}(X, E)$. Thus, since $p^{\prime}: E_{B}^{X} \rightarrow B$ is a pullback of the fibration $p_{\#}$, the fibration $p^{\prime}$ has a cross-section $s^{\prime}: B \rightarrow E_{B}^{X}$ defined by

$$
s^{\prime}(b)(x)=s(b) \quad(b \in B, x \in X),
$$

and the following diagram is commutative:

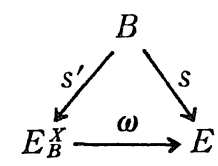

We need the following

Proposition 1.3. Let $p:\left(E^{\prime}, e_{0}^{\prime}\right) \rightarrow\left(E, e_{0}\right)$ and $k:\left(E, e_{0}\right) \rightarrow\left(B, b_{0}\right)$ be fibrations. Put $k^{\prime}=k \circ p$. Moreover, let $s:\left(B, b_{0}\right) \rightarrow\left(E, e_{0}\right)$ and $s^{\prime}:\left(B, b_{0}\right) \rightarrow\left(E^{\prime}, e_{0}^{\prime}\right)$ be crosssections with $p \circ s^{\prime}=s$ for the fibrations $k$ and $k^{\prime}$ respectively. When $k^{\prime \prime}$ is a map of $\left(X, x_{0}\right)$ to $\left(B, b_{0}\right)$, we have the following fibration 


$$
p_{\#}: \operatorname{map}_{0}\left(X, E^{\prime}\right)_{B} \rightarrow \operatorname{map}_{0}(X, E)_{B} .
$$

Proof can be done easily, so it is omitted.

\section{§2. Fibrations with Fibre $K(G, n)$}

Let $p: E \rightarrow B$ be a fibration with fibre $K(G, n)$ ( $G$ is an abelian group) over a $C W$ complex $B$. In the following, we denote by $B_{\infty}$ the classifying space for fibrations with fibre $K(G, n)$ and we shall investigate the loop space $\Omega \operatorname{map}_{0}\left(B, B_{\infty} ; k\right)[18]$ of $\operatorname{map}_{0}\left(B, B_{\infty} ; k\right)$, where $k$ is the classifying map of the above fibration.

For this purpose we prove the following

Theorem 2.1. Let $X$ be a $C W$ complex and let $\pi$ be an arbitrary group, then every path component of $\operatorname{map}_{0}(X, K(\pi, 1))$ is weakly contractible.

Proof. First we shall show $\pi_{i}\left(\operatorname{map}_{0}(X, K(\pi, 1) ; k)\right)=0$ for $i \geqq 2$, where $k$ is a map of $\left(X, x_{0}\right)$ to $\left(K(\pi, 1), y_{0}\right)$. Let $\bar{f}$ be a map of $\left(S^{i}, *\right)$ to $(\operatorname{map}(X, K(\pi, 1) ; k), k)$. Then we have its associated map $f: S^{i} \times X \rightarrow K(\pi, 1)$ with $f \mid * \times X=k$. The map $f:\left(S^{i} \times X, * \times x_{0}\right) \rightarrow\left(K(\pi, 1), y_{0}\right)$ induces the homomorphism $f_{*}$ :

$$
\pi_{1}\left(S^{i} \times X\right) \cong \pi_{1}(X) \rightarrow \pi_{1}(K(\pi, 1)) \cong \pi
$$

which is the same as the homomorphism $k_{*}: \pi_{1}(X) \rightarrow \pi$ induced by the map $k$.

Let $c$ be a map of $S^{i} \times X$ to $K(\pi, 1)$ defined by

$$
c(y, x)=k(x) \quad\left(x \in X, y \in S^{i}\right) .
$$

Then obviously $c$ induces the homomorphism $c_{*}: \pi_{1}\left(S^{i} \times X\right) \cong \pi_{1}(X) \rightarrow \pi$ which may be regarded as the homomorphism $k_{*}: \pi_{1}(X) \rightarrow \pi$. Therefore $f$ and $c$ are homotopic relative to $\left(*, x_{0}\right)$ [20]. This means that every map of $S^{i}$ to $\operatorname{map}(X, K(\pi, 1) ; k)$ is freely homotopic to the constant map $\bar{c}$ defined by $\bar{c}(y)=k$ for all $y \in S^{i}$. Therefore we have $\pi_{i}(\operatorname{map}(X, K(\pi, 1) ; k))=0$ for $i \geqq 2$.

Now, let $\omega$ be a map of $\operatorname{map}(X, K(\pi, 1) ; k)$ to $K(\pi, 1)$ defined by

$$
\omega(f)=f\left(x_{0}\right) \quad(f \in \operatorname{map}(X, K(\pi, 1) ; k)) .
$$

We get the following fibration:

$$
F \stackrel{j}{\longrightarrow} \operatorname{map}(X, K(\pi, 1) ; k) \stackrel{\omega}{\longrightarrow} K(\pi, 1),
$$

where $F$ is the fibre over $y_{0}$ which contains $\operatorname{map}_{0}(X, K(\pi, 1) ; k)$. Since $K(\pi, 1)$ is aspherical, it holds that

$$
\pi_{i}\left(\operatorname{map}_{0}(X, K(\pi, 1) ; k) \cong \pi_{\imath}(\operatorname{map}(X, K(\pi, 1) ; k))\right.
$$

for $i \geqq 2$. Consequently we have 
for $i \geqq 2$.

$$
\pi_{i}\left(\operatorname{map}_{0}(X, K(\pi, 1) ; k)\right)=0
$$

Next we note that the following lemma holds.

Lemma 2.2. $\pi_{1}\left(\operatorname{map}_{0}(X, K(\pi, 1) ; k)\right.$ is trivial.

A proof of this lemma is similarly performed to the proof of Lemma 3 in [3], so it is omitted.

Thus our proof of Theorem 2.1 is completed.

On the homotopy sequence of the fibration:

$$
F \stackrel{j}{\longrightarrow} \operatorname{map}(X, K(\pi, 1) ; k) \stackrel{\omega}{\longrightarrow} K(\pi, 1),
$$

we have the following

Corollary 2.3. Let $k_{*}: \pi_{1}(X) \rightarrow \pi$ be the homomorphism induced by the map $k$ and denote by $C_{k}$ the centralizer of $k_{*}\left(\pi_{1}(X)\right)$ in $\pi$. Then we have the following homotopy sequence of the above fibration

$$
1 \stackrel{j_{*}}{\longrightarrow} C_{k} \stackrel{\omega *}{\longrightarrow} \pi \stackrel{\partial}{\longrightarrow} R \longrightarrow 1
$$

where $C_{k}$ is isomorphic to $\pi_{1}(\operatorname{map}(X, K(\pi, 1) ; k)$ and $R$ is the subset of $\operatorname{Hom}\left(\pi_{1}(X), \pi\right)$ consisting of elements $\alpha^{-1} k_{*} \alpha(\alpha \in \pi)$.

Proof. We shall show in the following that the boundary $\partial: \pi_{1}(K(\pi, 1)) \cong$ $\pi \rightarrow \pi_{0}(F)$ is just given by

$$
\partial(\alpha)=\alpha^{-1} k_{*} \alpha,
$$

where $\pi_{0}(F)$ may be regarded as $R$. For a given element $\alpha$ of $\pi_{1}(K(\pi, 1))$, let $f:(I, \partial I) \rightarrow\left(K(\pi, 1), y_{0}\right)$ be a map representing $\alpha$ and let $\bar{F}:(I, 0) \rightarrow$ $(\operatorname{map}(X, K(\pi, 1) ; k), k)$ be a map such that $\omega \circ \bar{F}=f$. Then there exists the map $F: X \times I \rightarrow K(\pi, 1)$ associated with $\bar{F}$ such that

$$
\begin{aligned}
& F(x, 0)=k(x) \\
& F\left(x_{0}, t\right)=f(t) \quad(x \in X, t \in I) .
\end{aligned}
$$

If we put $F(x, 1)=k^{\prime}(x)$ for $x \in X$, we easily see

$$
k_{*}^{\prime}=\alpha^{-1} k_{*} \alpha .
$$

Namely, for every $\lambda$ of $\pi_{1}(X)$ it holds that

$$
k_{*}^{\prime}(\lambda)=\alpha^{-1} k_{*}(\lambda) \alpha .
$$

Thus we see $\partial(\alpha)=\alpha^{-1} k_{*} \alpha$.

Furthermore we can easily see that

$$
\partial(\alpha \beta)=\beta^{-1} \partial(\alpha) \beta .
$$


By using this equality we find that $\partial^{-1}\left(k_{*}\right)$ is a subgroup of $\pi_{1}(K(\pi, 1))$ which is the centralizer of $k_{*}\left(\pi_{1}(X)\right)$ in $\pi_{1}(K(\pi, 1))$. By Theorem $2.1 \operatorname{map}_{0}(X, K(\pi, 1) ; k)$ is weakly contractible. Therefore we have

$$
\pi_{1}(\operatorname{map}(X, K(\pi, 1)) ; k) \cong C_{k} \text {. }
$$

Note that this is known in Lemma 2 of Gottlieb [4].

Now, for the classifying space $B_{\infty}$ we have the following fibration:

$$
K(G, n+1) \stackrel{i_{0}}{\longrightarrow} B_{\infty} \stackrel{p_{0}}{\longrightarrow} K(\operatorname{Aut}(G), 1) .
$$

Let $X$ be a $C W$ complex, then we have the following fibration:

$$
F \longrightarrow \operatorname{map}_{0}\left(X, B_{\infty} ; k\right) \stackrel{p_{0 \#}}{\longrightarrow} \operatorname{map}_{0}\left(X, K(\operatorname{Aut}(G), 1) ; p_{0} \circ k\right),
$$

where $F$ is the fibre over $p_{0} \circ k$.

Proposition 2.4. With the above notations, we have

$$
\Omega \operatorname{map}_{0}\left(X, B_{\infty} ; k\right) \underset{w}{\widetilde{w}} \Omega F,
$$

where $Y \underset{w}{\widetilde{w}} Z$ means that $Y$ has the same weak homotopy type as $Z$.

Proof. By Theorem $2.1 \operatorname{map}_{0}\left(X, K(\operatorname{Aut}(G), 1) ; p_{0} \circ k\right)$ is weakly contractible. Therefore $F$ is weakly homotopy equivalent to $\operatorname{map}_{0}\left(X, B_{\infty} ; k\right)$. Thus we have

$$
\Omega F \underset{w}{\widetilde{w}} \Omega \operatorname{map}_{0}\left(X, B_{\infty} ; k\right) .
$$

\section{$\S 3$. The Classifying Space for Fibration with Fibre $K(G, n)$}

Let $G$ be an abelian group and $\operatorname{Aut}(G)$ its group of automorphisms. Then there exists an Eilenberg-MacLane complex $K(G, n+1)(n \geqq 0)$ which is a topological abelian group [7] and on which $\operatorname{Aut}(G)$ acts on the left by base point preserving cellular homeomorphisms ((5.2.5) Lemma in $[1], 1.2$. Lemma in [12]), here the base point is the identity element of $K(G, n+1)$. Let $W$ be a complex $K(\operatorname{Aut}(G), 1)$ and $\widetilde{W}$ its universal covering complex. Then $\operatorname{Aut}(G)$ acts on $\widetilde{W}$ freely and cellularly on the left. Thus $\operatorname{Aut}(G)$ acts on $\widetilde{W} \times K(G, n+1)$ diagonally. We denote by $L(G, n+1)$ the quotient

$$
(\widetilde{W} \times K(G, n+1)) / \operatorname{Aut}(G) .
$$

The projection of $\widetilde{W} \times K(G, n+1)$ onto $\widetilde{W}$ induces a map $p_{0}: L(G, n+1) \rightarrow$ $W=K(\operatorname{Aut}(G), 1)$ which is a fibre bundle with fibre $K(G, n+1)$ and with structure group $\operatorname{Aut}(G)$. Each fibre of this fibration is a topological abelian group isomorphic to $K(G, n+1)$ and there exists a canonical cross-section $3_{0}: \mathrm{IT}^{-} \rightarrow$ $L(G, n+1)$. It is well known that $L(G, n+1)$ is a classifying space $B_{\varsigma}$ for 
fibrations with fibre $K(G, n)[5,10]$.

Let $X$ be a $C W$ complex and let $k:\left(X, x_{0}\right) \rightarrow\left(W, w_{0}\right)=\left(K(\operatorname{Aut}(G), 1), w_{0}\right)$ be a space over $\left(W, w_{0}\right)$. Then we define a multiplication in the space $\operatorname{map}_{0}(X, L(G, n+1))_{W}$, where the fibration $p_{0}:\left(L(G, n+1), l_{0}\right) \rightarrow\left(W, w_{0}\right)$ is the space over $\left(W, w_{0}\right)$ and the canonical cross-section $s_{0}:\left(W, w_{0}\right) \rightarrow\left(L(G, n+1), l_{0}\right)$ is equipped.

Let $f$ and $g$ be any elements of $\operatorname{map}_{0}(X, L(G, n+1))_{W}$, then we define multiplication $f \cdot g$ of $f$ and $g$ by

$$
(f \cdot g)(x)=f(x) g(x) \quad(x \in X),
$$

because both $f(x)$ and $g(x)$ are contained in the fibre $p^{-1}(k(x))$ over $k(x)$. We can easily see that

$$
f \cdot g \in \operatorname{map}_{0}(X, L(G, n+1))_{W} .
$$

Thus we obtain the following

Proposition 3.1. Let $X$ be a $C W$ complex and $k:\left(X, x_{0}\right) \rightarrow\left(W, w_{0}\right)=$ $\left(K(\operatorname{Aut}(G), 1), w_{0}\right)$ be a space over $\left(W, w_{0}\right)$. Then, with respect to the multiplication defined above $\operatorname{map}_{0}(X, L(G, n+1))_{W}$ is a topological abelian group.

Proof is easily done, so it is omitted.

We observed that for $n \geqq 0$ there exists the following fibre bundle with structure group $\operatorname{Aut}(G)$ :

$$
K(G, n+1) \stackrel{i_{0}}{\longrightarrow} L(G, n+1) \stackrel{p_{0}}{\longrightarrow} K(\operatorname{Aut}(G), 1)=W .
$$

In the following we abbreviate this fibration by the fibration $p_{0}: L \rightarrow W$. So we have the space $p_{0}:\left(L, l_{0}\right) \rightarrow\left(W, w_{0}\right)$ over $\left(W, w_{0}\right)$ and the canonical cross-section $s_{0}:\left(W, w_{0}\right) \rightarrow\left(L, l_{0}\right)$.

In Remark 1.2 , if we replace $\left(X, x_{0}\right)$ by $\left(S^{i}, *\right)(i \geqq 0)$ and replace a fibration $p: E \rightarrow B$ by the fibration $p_{0}: L \rightarrow W$, then we have the fibration $\omega: L S_{W}^{i} \rightarrow L$, and the cross-section $s^{\prime}:\left(W, w_{0}\right) \rightarrow\left(L_{W}^{2}, c_{l_{0}}\right)$ for the fibration $p_{0} \circ \omega: L S_{W}^{2} \rightarrow W$, where $c_{l_{0}}$ denotes the constant map of $S^{i}$ to $l_{0}$. Also we have the following commutative diagram

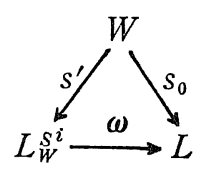

Let us denote $\omega^{-1}\left(s_{0}(W)\right)$ by $\bar{\Omega}^{i} L$ for $n+1>i \geqq 0$, then we have a fibration $((2.5)$ in [1], the proof of Lemma 1.2 in [2], [6]):

$$
K(G, n+1-i) \longrightarrow \bar{\Omega}^{i} L \stackrel{p_{0}}{\longrightarrow} W,
$$

such that $\bar{\Omega}^{i} L$ may be regarded as a space $L(G, n+1-i)$. 
With these notations we have the following

Lemma 3.2. Let $X$ be a CW complex, $k$ be a fixed map of $\left(X, x_{0}\right) \rightarrow\left(L, l_{0}\right)$ and $p_{0} \circ k=k^{\prime}:\left(X, x_{0}\right) \rightarrow\left(W, w_{0}\right)$ be a space over $\left(W, w_{0}\right)$. We have the following isomorphisms:

$$
\pi_{\imath}\left(\operatorname{map}_{0}(X, L)_{W}, s_{0} \circ k^{\prime}\right) \cong\left[X, \bar{\Omega}^{i} L\right]_{W}^{0} \cong H^{n+1-\imath}\left(X, x_{0} ; G\right),
$$

where $\left[X, \bar{\Omega}^{i} L\right]_{\mathrm{W}}^{0}$ denotes the pointed homotopy classes over $\mathrm{W}$ of maps from $\left(X, x_{0}\right)$ to $\left(\bar{\Omega}^{i} L, c_{l_{0}}\right)$ and the cohomology is taken with local coefficients classified by the map $k^{\prime}: X \rightarrow W$.

Proof. Let $\bar{f}$ be a map of $\left(S^{i}, *\right)$ to $\left(\operatorname{map}_{0}(X, L)_{W}, s_{0} \circ k^{\prime}\right)$. Then we have its associated map $f: S^{i} \times\left(X, x_{0}\right) \rightarrow\left(L, l_{0}\right)$ with $f \mid * \times X=s_{0} \circ k^{\prime}$ such that the following diagram is commutative

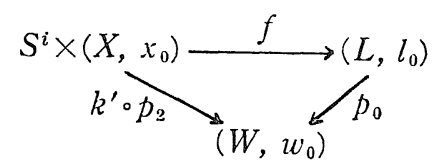

where $p_{2}$ is the projection of $S^{i} \times\left(X, x_{0}\right)$ onto $\left(X, x_{0}\right)$.

Furthermore $f$ corresponds to the map $\tilde{f}$ of $\left(X, x_{0}\right)$ to $\left(L_{V W}^{2}, c_{l_{0}}\right)$ defined by

$$
\tilde{f}(x)(y)=f(y, x) \quad\left(x \in X, y \in S^{i}\right) .
$$

In fact, since for $y \in S^{i}$ we have

$$
p_{0}(\tilde{f}(x)(y))=p_{0} \circ f(y, x)=k^{\prime}(x) \quad(x \in X),
$$

we see that $\tilde{f}(x)$ is an element of $L_{W}^{S^{2}}$ for $x \in X$. By using

$$
\omega \circ \tilde{f}(x)=\tilde{f}(x)(*)=s_{0} \circ k^{\prime}(x) \quad(x \in X),
$$

we can easily see that $\tilde{f}$ is a map of $\left(X, x_{0}\right)$ to $\left(\bar{\Omega}^{i} L, c_{l_{0}}\right)$ and the following diagram is commutative:

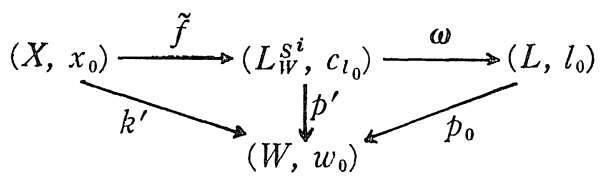

where $p^{\prime}$ denotes $p_{0} \circ \omega$.

Similarly a homotopy $\bar{H}:\left(S^{i}, *\right) \times I \rightarrow\left(\operatorname{map}_{0}(X, L)_{W}, s_{0} \circ k^{\prime}\right)$ corresponds to a homotopy $\tilde{H}:\left(X, x_{0}\right) \times I \rightarrow\left(\bar{\Omega}^{i} L, c_{l_{0}}\right)$. We can easily see that this correspondence induces the bijection

$$
\pi_{\imath}\left(\operatorname{map}_{0}(X, L)_{W}, s_{0} \circ k^{\prime}\right) \cong\left[X, \bar{\Omega}^{i} L\right]_{W}^{0} .
$$

Since $\bar{\Omega}^{i} L$ is a $L(G, n+1-i)$, we have the following isomorphisms ((5.2.4) 
Theorem in [1], 3.1 Definition in [10], (6.13) Theorem in Chapter VI of [20])

$$
\left[X, \bar{\Omega}^{2} L\right]_{W}^{0} \cong[X, L(G, n+1-i)]_{W}^{0} \cong H^{n+1-i}\left(X, x_{0} ; G\right),
$$

where $H^{n+1-2}\left(X, x_{0} ; G\right)$ is the cohomology group with local coefficients classified by the map $k^{\prime}$.

From this lemma we get the following

Theorem 3.3. Let $X$ be a $C W$ complex, $k$ be a fixed map of $\left(X, x_{0}\right)$ to $\left(L, l_{0}\right)$ and $p_{0} \circ k=k^{\prime}:\left(X, x_{0}\right) \rightarrow\left(W, w_{0}\right)$ be a space over $\left(W, w_{0}\right)$. Then $\operatorname{map}_{0}(X, L)_{W}$ has the same weak homotopy type as

$$
H^{n+1}\left(X, x_{0} ; G\right) \times \prod_{i=1}^{n} K\left(H^{n+1-i}\left(X, x_{0} ; G\right), i\right),
$$

where the cohomology is taken with local coefficients classified by the map $k^{\prime}: X \rightarrow W$.

Proof. Notice that $[X, L]_{W}^{0}$ is isomorphic to the group $H^{n+1}\left(X, x_{0} ; G\right)$. Thus by using Proposition 3.1 and the theorem of J. C. Moore [8] we have

$$
\operatorname{map}_{0}(X, L)_{W} \underset{w}{\widetilde{w}} H^{n+1}\left(X, x_{0} ; G\right) \times \prod_{i=1}^{n} K\left(H^{n+1-i}\left(X, x_{0} ; G\right), i\right) .
$$

\section{§4. Main Results}

Recall the fibration:

$$
F \longrightarrow \operatorname{map}_{0}\left(X, B_{\infty} ; k\right) \stackrel{p_{0 \#}}{\longrightarrow} \operatorname{map}_{0}\left(X, K(\operatorname{Aut}(G), 1) ; p_{0} \circ k\right),
$$

where $F$ is the fibre over $p_{0} \circ k=k^{\prime}$. In the following we shall investigate the loop space $\Omega F$ of $F$.

Since the fibration ${\phi_{0}}_{0}: L \rightarrow W$ has a canonical cross-section $s_{0}:\left(W, w_{0}\right) \rightarrow\left(L, l_{0}\right)$, by Proposition 1.1 and Remark 1.2 we have the following commutative diagram:

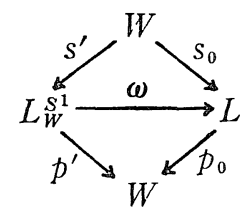

where $p^{\prime}=p_{0} \circ \omega$ and $s_{0}=\omega \circ s^{\prime}$. Let $k$ be a map of a given $C W$ complex $\left(X, x_{0}\right)$ to $\left(L, l_{0}\right)$. If we put $p_{0} \circ k=k^{\prime}$, by Proposition 1.3 we have the following fibration $\omega_{\ddagger}$ :

$$
\operatorname{map}_{0}\left(X, L_{W}^{S^{1}}\right)_{W} \longrightarrow \operatorname{map}_{0}(X, L)_{W}
$$

On a relation between this fibration and $\Omega F$, we have the following

Lemma 4.1. $\Omega F$ is homeomorphic to the fibre $\omega_{\equiv}^{-1}(k)$ over $k$. 
Proof. Let $\bar{f}$ be a map of $\left(S^{1}, *\right)$ to $(F, k)$. Then we have its associated map $f: S^{1} \times\left(X, x_{0}\right) \rightarrow\left(L, l_{0}\right)$ such that

$$
\begin{gathered}
f(*, x)=k(x), \\
p_{0} \circ f(t, x)=k^{\prime}(x) \quad\left(=p_{0} \circ k(x)\right) \quad\left(t \in S^{1}, x \in X\right) .
\end{gathered}
$$

We may define a map $\tilde{f}$ of $\left(X, x_{0}\right)$ to $\left(L_{W}^{1}, c_{l_{0}}\right)$ by $\tilde{f}(x)(t)=f(t, x)$ for $t \in S^{1}$ and $x \in X$, because

$$
\begin{aligned}
p_{0} \circ \omega \circ \tilde{f}(x) & =p_{0}(\tilde{f}(x)(*))=p_{0} \circ f(*, x) \\
& =p_{0} \circ k(x)=k^{\prime}(x) \quad(x \in X) .
\end{aligned}
$$

Thus we see that $\tilde{f}:\left(X, x_{0}\right) \rightarrow\left(L_{W}^{S^{1}}, c_{l_{0}}\right)$ is a map over $\left(W, w_{0}\right)$. Also we see that $\omega_{\#}(\tilde{f})=k$, that is, $\tilde{f}$ is an element of a fibre $\omega_{\#}^{-1}(k)$ of the fibration $\omega_{\#}$ : $\operatorname{map}_{0}\left(X, L_{W}^{1}\right)_{W} \rightarrow \operatorname{map}_{0}(X, L)_{W}$. As easily seen, this correspondence gives rise to a homeomorphism of $\Omega F$ onto the fibre $\omega_{\#}^{-1}(k)$ over $k$.

Now, for any $C W$ complex $K$ we have the following commutative diagram:

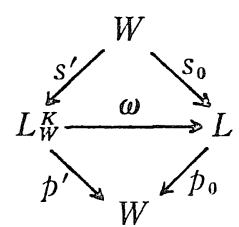

We denote by $L_{W}^{K} \times_{W} L_{W}^{K}$ the fibred product of the fibration $p^{\prime}: L_{W}^{K} \rightarrow W$ and itself. It should be noted that there exists a map $\mu$ of $L_{W}^{K} \succ_{W} L_{W}^{K}$ to $L_{W}^{K}$ defined by

$$
\mu(f, g)(y)=f(y) g(y) \quad(y \in K),
$$

because $f(y)$ and $g(y)$ for every $y \in K$ are contained in the same fibre $p_{0}^{-1}\left(p^{\prime}(f)\right)$ $=p_{0}^{-1}\left(p^{\prime}(g)\right)$. Especially we have a map $\mu$ of $L_{W}^{S^{1}} \times_{W} L_{W}^{S^{1}}$ to $L_{W V}^{S^{1}}$. By using this multiplication of $L_{W}^{S 1}$ we have the following

Proposition 4.2. Let $X$ be a $C W$ complex, $k$ be a fixed map of $\left(X, x_{0}\right)$ to $\left(L, l_{0}\right)$ and $p_{0} \circ k=k^{\prime}:\left(X, x_{0}\right) \rightarrow\left(W, w_{0}\right)$ be a space over $\left(W, w_{0}\right)$. Then $\operatorname{map}_{0}\left(X, L_{W}^{S^{1}}\right)_{W}$ is a topological abelian group and the projection $\omega_{\#}: \operatorname{map}_{0}\left(X, L_{W}^{S}\right)_{W} \rightarrow \operatorname{map}_{0}(X, L)_{W}$ is an epimorphism of topological groups.

Proof. For any elements $\tilde{f}$ and $\tilde{g}$ of $\operatorname{map}_{0}\left(X, L_{W}^{S^{1}}\right)_{W}$ the multiplication $\tilde{f} \cdot \tilde{g}$ is defined by

$$
(\tilde{f} \cdot \tilde{g})(x)=\tilde{f}(x) \cdot \tilde{g}(x) \quad(x \in X),
$$

where $\tilde{f}(x) \cdot \tilde{g}(x)$ means $\mu(\tilde{f}(x), \tilde{g}(x))$. Note that $\mu(\tilde{f}(x), \tilde{g}(x))$ is well defined, because $(\tilde{f}(x), \tilde{g}(x))$ is an element of $L_{W}^{S^{1}} \times_{W} L_{W}^{S^{1}}$ by

$$
p^{\prime}(\tilde{f}(x))=p^{\prime}(\tilde{g}(x))=k^{\prime}(x) \quad(x \in X) .
$$


Since $p^{\prime}(\tilde{f} \circ \tilde{g})(x)=p^{\prime} \circ \tilde{f}(x)=k^{\prime}(x)$, we see that $\tilde{f} \cdot \tilde{g}$ is an element of $\operatorname{map}_{0}\left(X, L_{W}^{S^{1}}\right)_{W}$.

We can easily prove that with respect to this multiplication $\operatorname{map}_{0}\left(X, L_{W}^{S^{1}}\right)_{W^{r}}$ is a topological abelian group with the identity element $s^{\prime} \circ k^{\prime}$.

Next we shall show that $\omega_{\#}$ is a homomorphism of $\operatorname{map}_{0}\left(X, L_{W^{\prime}}^{S^{1}}\right)_{W^{\prime}}$ onto the group $\operatorname{map}_{0}(X, L)_{W r}$ Let $\tilde{f}$ and $\tilde{g}$ be any elements of $\operatorname{map}_{0}\left(X, L_{W}^{S^{1}}\right)_{W}$. Then we have for $x \in X$

$$
\begin{aligned}
\omega_{\#}(\tilde{f} \cdot \tilde{g})(x) & =(\tilde{f} \cdot \tilde{g})(x)(*)=(\tilde{f}(x) \cdot \tilde{g}(x))(*) \\
& =(\tilde{f}(x)(*))(\tilde{g}(x)(*)) \\
& =\left(\omega_{\#}(\tilde{f})(x)\right)\left(\omega_{\#}(\tilde{g})(x)\right) \\
& =\left(\omega_{\#}(f) \cdot \omega_{\#}(g)\right)(x) .
\end{aligned}
$$

Thus we have $\omega_{\#}(\tilde{f} \cdot \tilde{g})=\omega_{\#}(\tilde{f}) \cdot \omega_{\#}(\tilde{g})$.

Define a map $\sigma: L \rightarrow L_{W^{\prime}}^{S}$ as follows :

$$
\sigma(l)(t)=l \quad\left(l \in L, t \in S^{1}\right) .
$$

Then we see easily that $\sigma$ is a map over $\left(W, w_{0}\right)$ and a cross-section for the fibration $\omega: L S_{W}^{1} \rightarrow L$. So $\sigma$ induces the map $\sigma_{\#}: \operatorname{map}_{0}(X, L)_{W} \rightarrow \operatorname{map}_{0}\left(X, L_{W}^{S^{1}}\right)_{W}$ which is a cross-section for the fibration $\omega_{\#}: \operatorname{map}_{0}\left(X, L_{W}^{S^{1}}\right)_{W} \rightarrow \operatorname{map}_{0}(X, L)_{W}$. Therefore we see that $\omega_{\#}$ is surjective.

We can easily see $\omega_{\#}^{-1}\left(s_{0} \circ k^{\prime}\right)=\operatorname{map}_{0}(X, \bar{\Omega} L)_{W^{r}}$. Hence the following is an immediate consequence of Proposition 4.2.

Corollary 4.3。 Ker $\omega_{\#}=\omega_{\#}^{-1}\left(s_{0} \circ k^{\prime}\right)$ is homeomorphic to the fibre $\omega_{\ddagger}^{-1}(k)$ over $k$. In other words,

$$
\omega_{\#}^{-1}(k) \cong \operatorname{map}_{0}(X, \bar{\Omega} L)_{W}=\operatorname{map}_{0}(X, L(G, n))_{W} .
$$

Now, let $p: E \rightarrow B$ be a fibration with fibre $F=K(G, n)(n>1)$ over a $C W$ complex $B$. In $[18,19]$ the following result is shown for a simply connected $B$ :

$$
\begin{aligned}
\mathcal{G}(E \bmod F) & \underset{\widetilde{w}}{\widetilde{m_{a p}}} \operatorname{map}_{0}(B, K(G, n)) \\
& \underset{\bar{w}}{\widetilde{N}} H^{n}(B, G) \times \prod_{i=1}^{n-1} K\left(H^{n-i}(B, G), i\right),
\end{aligned}
$$

where $\mathcal{G}(E \bmod F)$ is the space of self fibre homotopy equivalences of $E$ leaving a fibre $p^{-1}\left(b_{0}\right)=F$ fixed. Here without assuming that $B$ is simply connected, we have a following generalization of this result which follows from our previous Proposition 2.4, Lemma 4.1, Corollary 4.3 and Theorem 3.3.

Theorem 4.4. Let $p: E \rightarrow B$ be a fibration with fibre $F=K(G, n)(n>1)$ such that $B$ is a CW complex. Then if we denote by $k:\left(B, b_{0}\right) \rightarrow\left(L(G, n+1), l_{0}\right) a$ corresponding map to the fibration: $F \stackrel{i}{\longrightarrow} E \stackrel{p}{\longrightarrow} B$, we have 


$$
\begin{aligned}
\mathcal{G}(E \bmod F) & \underset{w}{\widetilde{w}} \operatorname{map}_{0}(B, L(G, n))_{W} \\
& \underset{w}{\widetilde{w}} H^{n}(B, G) \times \prod_{\imath=1}^{n-1} K\left(H^{n-i}\left(B, b_{0} ; G\right), i\right)
\end{aligned}
$$

where the cohomology is taken with local coefficients classified by the map $p_{0} \circ k: B \rightarrow K(\operatorname{Aut}(G), 1)\left(p_{0}\right.$ is the projection of $L(G, n+1)$ to $K(\operatorname{Aut}(G), 1)$ ).

Now, we quote the following two theorems $[18,19]$, where $G_{0}(X)$ denotes the space of self homotopy equivalences of a $C W \operatorname{complex}\left(X, x_{0}\right)$.

Theorem 4.5. Let $E$ and $B$ be $C W$ complexes and let $p: E \rightarrow B$ be a fibration with fibre $F$. For a given $n>1$ if $F$ is $(n-1)$-connected and $\pi_{2}(B)=0$ for every $i \geqq n$, then we have the following fibration:

$$
\mathcal{G}(E \bmod F) \longrightarrow G_{0}(E) \stackrel{\rho}{\longrightarrow} G_{0}(B) \times G_{0}(F) .
$$

Theorem 4.6. Under the hypothesis of Theorem 4.5, the image of $\rho: G_{0}(E)$ $\rightarrow G_{0}(B) \times G_{0}(F)$ is just the union of the path components in $G_{0}(B) \times G_{0}(F)$ each of which contains $(g, h)$ satisfying

$$
\left[\chi_{\infty}(h)\right] \cdot[k]=[k] \cdot[g],
$$

where $\chi_{\infty}(h)$ is a self map of $\left(B_{\infty}, b_{\infty}\right)$ and $k:\left(B, b_{0}\right) \rightarrow\left(B_{\infty}, b_{\infty}\right)$ is a corresponding map to the fibration: $F \stackrel{i}{\longrightarrow} E \stackrel{p}{\longrightarrow} B$.

Let $\varepsilon(X)$ denote the group $\pi_{0}\left(G_{0}(X)\right)$ for a $C W$ complex $X$. Since $G_{0}(K(\pi, n))$ $\widetilde{\bar{w}} \operatorname{Aut}(\pi)$ for $n \geqq 1$, by Theorem $4.4,4.5$ and 4.6 we have the following theorem which is a generalization of Theorem 10 in [18].

Theorem 4.7. For given $1 \leqq m<n$, let

$$
F=K(G, n) \stackrel{i}{\longrightarrow} E \stackrel{p}{\longrightarrow} K(\pi, m)=B
$$

be a fibration with a corresponding map $k:\left(B, b_{0}\right) \rightarrow\left(L(G, n+1), l_{0}\right)$. Then we have

$$
G_{0}(E) \underset{w}{\widetilde{w}} R \times H^{n}(B, G) \times \prod_{i=1}^{n-1} K\left(H^{n-i}\left(B, b_{0} ; G\right), i\right),
$$

where $R$ is the subgroup of $\operatorname{Aut}(\pi) \times \operatorname{Aut}(G)=\varepsilon(B) \times \varepsilon(F)$ consisting of $([g],[h])$ with

$$
\left[\chi_{\infty}(h)\right] \cdot[k]=[k] \cdot[g],
$$

and the cohomology is taken with local coefficients classified by the map $p_{0} \circ k$ : $B \rightarrow K(\operatorname{Aut}(G), 1)=W\left(p_{0}\right.$ is the projection of $L(G, n+1)$ to $K(\operatorname{Aut}(G), 1)$.

Note that the map $\rho: G_{0}(E) \rightarrow G_{0}(B) \times G_{0}(F)$ induces the homomorphism $\rho_{*}$ of $\varepsilon(E)$ into $\varepsilon(B) \times \varepsilon(F)$, that the image of $\rho_{*}$ is just $R$ in Theorem 4.7 and that 
the kernel of $\rho_{*}$ may be regarded as $H^{n}(B, G)$. Thus, as a corollary of Theorem 4.7 we have the following theorem $[9,11,15]$.

Theorem 4.8. Under the same hypothesis of Theorem 4.7 there exists the following exact sequence

$$
1 \longrightarrow H^{n}(B, G) \longrightarrow \varepsilon(E) \longrightarrow R \longrightarrow 1,
$$

where $R$ is the same group as the group stated in Theorem 4.7 and the cohomology is taken with local coefficients given by the map $p_{0} \circ k: B \rightarrow K(\operatorname{Aut}(G), 1)$.

\section{References}

[1] Baues, H. J., Obstruction theory on homotopy classification of maps, Lecture Notes in Math., 628, Springer-Verlag, 1977.

[2] Dror, E. and A. Zabrodsky, Unipotency and nilpotency in homotopy equivalences, Topology, 18 (1979), 187-197.

[3] Gottlieb, D.H., A certain subgroup of the fundamental group, Amer. J. Math., 87 (1965), 840-846.

[4] - Covering transformations and universal fibration, Illinois J. Math., 13 (1969), 432-437.

[5] Gitler, S., Cohomology operations with local coefficients, Amer. J. Math., 85 (1963), $156-188$.

[6] McClendon, J.F., On stable fibre space obstructions, Pacific J. Math., 36 (1971), 439-445.

[7] Milgram, R., The bar construction and abelian H-spaces, Illinois J. Math., 11 (1967), 242-250.

[8] Moore, J.C., Seminar on algebraic homotopy theory, Princeton, 1956 (mimeographed notes).

[9] Nomura, Y., Homotopy equivalences in a principal fibre space, Math. Z., 92 (1966), $380-388$.

[10] Robinson, C. A., Moore-Postnikov systems for non-simple fibrations, Illinois J. Math., 16 (1972), 234-242.

[11] Shih, W., On the group $\varepsilon[X]$ of homotopy equivalence maps, Bull. Amer. Math. Soc., 70 (1964), 361-365.

[12] Siegel, J., Higher order cohomology operations in local coefficients theory, Amer.J. Math., 89 (1967), 909-931.

[13] Steenrod, N.E., A convenient category of topological spaces, Michigan Math.J., 14 (1967), 133-152.

[14] Strøm, A., Note on cofibrations II, Math. Scad., 22 (1968), 130-142.

[15] Tsukiyama, K., Self-homotopy-equivalences of a space with two nonvanishing homotopy groups, Proc. Amer. Math. Soc., 79 (1980), 134-138.

[16] Yamanoshita, T., On the spaces of self homotopy equivalences of certain $\mathrm{CW}$ complexes, Proc. Japan Acad., 60A (1984), 229-231.

[17] - On the spaces of self homotopy equivalences of certain $\mathrm{CW}$ complexes, J. Math. Soc. Japan, to appear.

[18] - On the spaces of self homotopy equivalences for fibre spaces, Proc. Japan Acad., 61A (1985), 15-18.

[19] — On the spaces of self homotopy equivalences for fibre spaces I, in preparation.

[20] Whitehead, G.W., Elements of Homotopy Theory, Graduate Texts in Math., 61, Springer-Verlag, New York, Heidelberg, Berlin, 1978. 